\title{
EC absorption efficiency in ITER at one-third nominal magnetic field strength
}

\author{
Daniele Micheletti ${ }^{*}$, Daniela Farina, and Lorenzo Figini \\ Istituto di Fisica del Plasma - CNR, 20125 Milano, Italy
}

\begin{abstract}
During the first phase of the ITER Pre-Fusion Power Operations (PFPO-1), the Electron Cyclotron Radio Frequency system will be the main heating and current drive source [1]. Evaluations of the L-H power threshold [2] have shown that H-mode is unlikely to be achieved in PFPO-1 scenarios at halffield $\left(\mathrm{B}_{0}=2.65 \mathrm{~T}\right)$. For this reason, the use of one-third field scenarios in an early phase might help to access $\mathrm{H}$-mode. This means that the EC Heating (ECH) system, with gyrotrons operating at $170 \mathrm{GHz}$, would then rely on $n=3$ harmonic resonance. Using a lower frequency, namely $104 \mathrm{GHz}$, could allow the exploitation of $\mathrm{n}=2$ harmonic resonance, improving heating efficiency and facilitating EC-assisted breakdown which has not been demonstrated yet for $\mathrm{n}=3$ harmonic. This work presents a preliminary evaluation of the performances of an EC system using the two frequencies in a $\mathrm{B}_{0}=1.8 \mathrm{~T}$ scenario (obtained by adequately rescaling a reference half-field scenario [3]) via a parametric scan of density/temperature profiles, to cover the range of possible values expected to occur during the operations and provide an estimation of the parameter space region where the use of each frequency allows a good absorption efficiency.
\end{abstract}

\section{Introduction}

The initial phase of the ITER research plan, after the First Plasma and before the D and DT operations, consists of non-activated $\mathrm{H} / \mathrm{He}$ plasmas in which the Lto-H-mode transition power thresholds will be investigated [1]. In the first part of this phase, named Pre-Fusion Power Operation 1 (PFPO-1), the Heating and Current Drive system (H\&CD) will consist of a 20 MW Electron Cyclotron Radio Frequency (ECRF) system, and a possible $10 \mathrm{MW}$ Ion Cyclotron RF system. During the PFPO- 1 the magnetic field will start at half the nominal field strength, that is $\mathrm{B}_{0}=2.65 \mathrm{~T}$, and then proceed towards full-field $\left(\mathrm{B}_{0}=5.3 \mathrm{~T}\right)$. It has been shown [2] that $\mathrm{H}$-mode access at half-field in this scenario is unlikely due to the high power threshold. For this reason, a one-third field scenario $\left(\mathrm{B}_{0}=1.8 \mathrm{~T}\right)$ has been proposed, since it could help to overcome the L-to$\mathrm{H}$ threshold and access $\mathrm{H}$-mode. The ITER gyrotrons will operate at $f=170 \mathrm{GHz}$, taking advantage of $n=1$ harmonic resonance at the nominal field and $n=2$ harmonic resonance at half-field. Using a magnetic field at one-third strength means that the heating system would rely on the lower efficiency of $n=3$ harmonic resonance absorption. Using dual-frequency gyrotrons working both at $170 \mathrm{GHz}$ and at a lower frequency, namely $104 \mathrm{GHz}$, would allow to exploit the $\mathrm{n}=2$ harmonic resonance absorption at $1.8 \mathrm{~T}$ and its higher absorption efficiency at the beginning of the plasma discharge, so to increase the temperature till the point at which the absorption of $170 \mathrm{GHz} \mathrm{EC}$ waves by the $\mathrm{n}=3$ harmonic reaches a safe level. Besides, $n=3$ harmonic
EC-assisted breakdown has not been achieved up to now.

The present work aims at presenting a preliminary evaluation of the performances of an EC system operating at the two frequencies $(170 / 104 \mathrm{GHz})$ in a onethird magnetic field scenario. The ITER ECRF system, the reference scenario and the modelling framework used for the analysis are briefly described in Section 2. The performances of the EC launchers for $f=170 \mathrm{GHz}$ are evaluated in Section 3 by calculating absorption efficiency and access to inner plasma region for a wide range of electron density $\left(\mathrm{n}_{\mathrm{e}}\right)$ and temperature $\left(\mathrm{T}_{\mathrm{e}}\right)$ values. The analysis is repeated for the lower frequency $\mathrm{f}$ $=104 \mathrm{GHz}$ in Section 4. The results of the analysis are summarised in Section 5 .

\section{Modelling framework}

\subsection{ITER ECRF system}

The ITER ECRF system consists of 5 launchers: 1 Equatorial Launcher and 4 Upper Launchers. The Equatorial Launcher (EL) includes 3 mirrors (TOP, MID, LOW), while each Upper Launchers (UL) includes 2 mirrors, Upper and Lower Steering Mirror (USM, LSM). The launchers are connected to 24 gyrotrons working at $\mathrm{f}=170 \mathrm{GHz}$, providing $1 \mathrm{MW}$ each $(0.83$ MW effectively injected into the plasma due to losses along the transmission line). During PFPO-1 all 24 gyrotrons will be available, for a total of $20 \mathrm{MW}$ injectable into the plasma. The gyrotrons output can be

\footnotetext{
*Corresponding author: micheletti@ifp.cnr.it
} 
split among the launchers: each of the EL mirrors can inject 8 beams into the plasma, while the UL mirrors uses 4 beams each.

\subsection{Plasma reference scenario}

The analysis presented in the following sections is based on the ITER half-field scenario $\left(\mathrm{B}_{0}=2.65 \mathrm{~T}, \mathrm{I}_{\mathrm{p}}=7.5\right.$ MA, He plasma, H-mode) [3] rescaled to one-third field $\left(\mathrm{B}_{0}=1.8 \mathrm{~T}, \mathrm{I}_{\mathrm{p}}=5 \mathrm{MA}\right)$, keeping constant safety factor $\mathrm{q}$ and Greenwald density fraction. Fig. 1 shows $T_{e}$ and $n_{e}$ profiles of the resulting scenario (the same scaling factor was used for both profiles). In the following sections, the scaled profiles will be referred to using their central plasma values, $\mathrm{T}_{\mathrm{e}, 0}=\mathrm{T}_{\mathrm{e}}(\rho=0)$ and $\mathrm{n}_{\mathrm{e}, 0}=\mathrm{n}_{\mathrm{e}}(\rho=0)$.

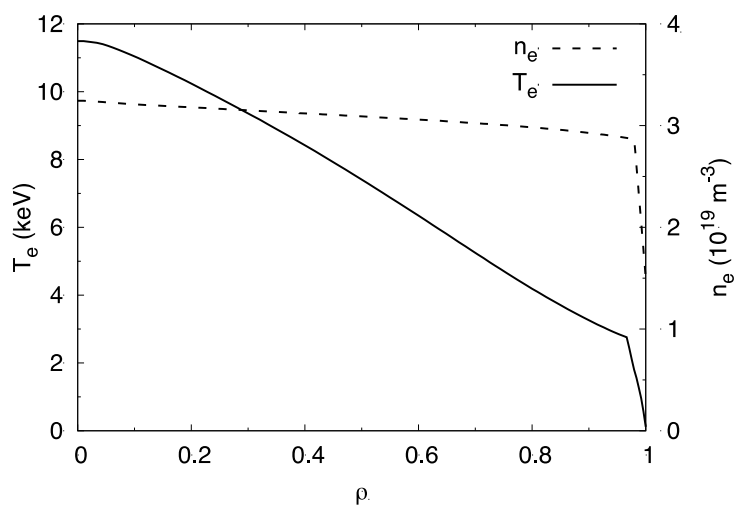

Fig. 1. $T_{e}$ and $n_{e}$ profiles for $B_{0}=1.8 T$ scenario.

\subsection{Simulations and optimal injection angle}

The ECH\&CD calculations have been carried out using the beam-tracing code GRAY [4], using a representative $1 \mathrm{MW}$ X-mode polarized beam for each mirror. Tables 1a,b list the mirror positions, the injection angles and the beam parameters at launch (width and radii of curvature along directions perpendicular to propagation) used.

Table 1a. Beam starting position and injection angles.

\begin{tabular}{ccccc} 
& $\boldsymbol{R}(\mathbf{m})$ & $\mathbf{z}(\mathbf{m})$ & $\boldsymbol{\alpha}\left({ }^{(\mathbf{})}\right.$ & $\boldsymbol{\beta}\left(^{\mathbf{}}\right)$ \\
\hline TOP & 9.394 & 1.192 & $-20 \div 15$ & -20 \\
\hline MID & 9.394 & 0.620 & $0 \div 35$ & 25 \\
\hline LOW & 9.394 & -0.004 & $-10 \div 25$ & 25 \\
\hline USM & 6.999 & 4.414 & $40 \div 65$ & 20 \\
\hline LSM & 7.054 & 4.178 & $30 \div 55$ & 20
\end{tabular}

Table 1b. Starting beam waist and radii of curvature.

\begin{tabular}{ccccc} 
& $\mathbf{w}_{\mathbf{x}}(\mathbf{c m})$ & $\mathbf{w}_{\mathbf{y}}(\mathbf{c m})$ & $\mathbf{R}_{\mathbf{x}}(\mathbf{c m})$ & $\mathbf{R}_{\mathbf{y}}(\mathbf{c m})$ \\
\hline TOP & 2.65 & 2.76 & -63.53 & -210.6 \\
\hline MID & 2.65 & 2.76 & -63.53 & -210.6 \\
\hline LOW & 2.65 & 2.76 & -63.53 & -210.6 \\
\hline USM & 5.05 & 5.05 & -318.6 & -318.6 \\
\hline LSM & 4.81 & 4.81 & -200.1 & -200.1 \\
\hline
\end{tabular}

The poloidal $(\alpha)$ and toroidal $(\beta)$ injection angles, defined from the cylindrical components of the wavevector at launch $\boldsymbol{k}=\left(k_{\mathrm{r}}, k_{\Phi}, k_{\mathrm{z}}\right)$ as

$$
\begin{aligned}
& \tan \alpha=k_{z} / k_{r} \\
& \sin \beta=k_{\Phi} /|\boldsymbol{k}|
\end{aligned}
$$

impact on the absorption efficiency. The values of $\beta$ correspond to the most recent design of the EC system [1], while $\alpha$ cover the full steering range available for each mirror. Fig. 3 shows an example of beam paths for $\mathrm{f}$ $=170 \mathrm{GHz}$. It can be noticed that both $n=3$ and $n=4$ harmonic are in the plasma, on HFS and LFS.

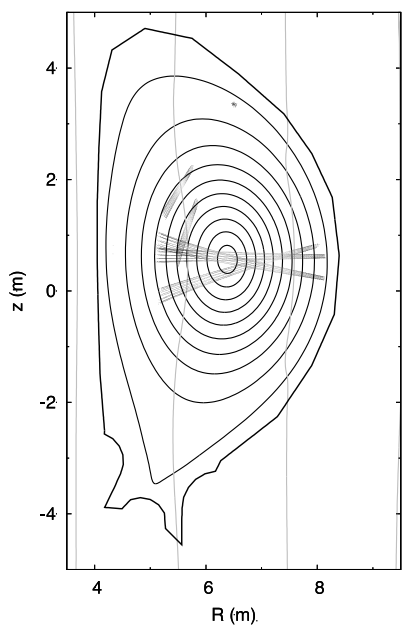

Fig. 2. Propagating $170 \mathrm{GHz}$ beams for UL and EL, with harmonic resonance locations ( $n=3,4$ inside the plasma).

To cover the range of possible values expected to occur during the discharge process in PFPO-1, $\mathrm{n}_{\mathrm{e}}$ and $\mathrm{T}_{\mathrm{e}}$ profiles have been varied by means of an independent parametric scaling, keeping constant $\mathrm{B}$ and $\mathrm{I}_{\mathrm{p}}$, as

$$
\begin{aligned}
& n_{e}^{\prime}(\rho)=f_{n} \cdot n_{e}(\rho) \\
& T_{e}^{\prime}(\rho)=f_{T} \cdot T_{e}(\rho)
\end{aligned}
$$

with the scaling factors $0.05 \leq f_{n}, f_{T} \leq 1$.

The optimal poloidal steering angle $\alpha_{\text {opt }}$, defined as the angle that maximizes the absorbed power fraction, has been determined for each mirror via a scan on the steering range for different $\mathrm{T}_{\mathrm{e}}, \mathrm{n}_{\mathrm{e}}$ scaling combinations, and has then been used throughout the subsequent analysis. As shown in Fig. 3, $\alpha_{\mathrm{opt}}$ is located at the extreme of the steering range corresponding to the beam pointing toward the centre of the plasma. This is true for every mirror and profile combination.

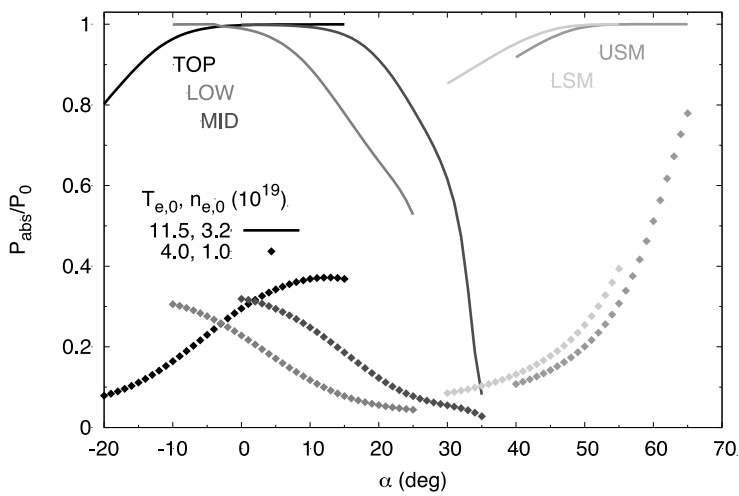

Fig. 3. Absorbed power fraction as a function of $\alpha$ for different $\mathrm{T}_{\mathrm{e}}, \mathrm{n}_{\mathrm{e}}$ scaling. 


\section{Performances at $170 \mathrm{GHz}$}

\subsection{Power absorption efficiency}

The analysis, as written in the previous section, has been performed for a wide range of temperature and density scaling factors. Fig. $4 a, b$ show the dependence of absorption efficiency on electron temperature and density for two mirrors, while in Fig. 5 corresponds to slices at constant $\mathrm{n}_{\mathrm{e}, 0}$ (for all mirrors). As expected, the efficiency increases more rapidly with $T_{e}$ than $n_{e}$ (optical thickness for $n=3$ harmonic proportional to $n_{e} T_{e}^{2}$ ). It can be seen that for MID (similar results obtained for TOP, LOW and LSM) a large fraction of the injected power is not absorbed at typical temperature values of an ohmic plasma.

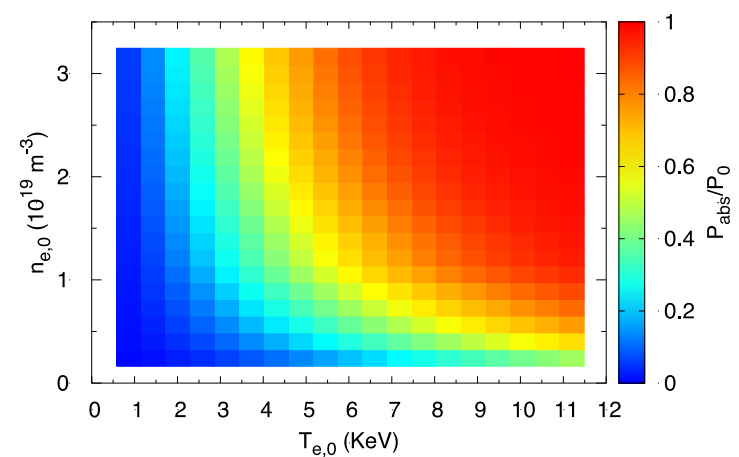

Fig. 4a. Absorption efficiency as a function of $T_{e, 0}, n_{e, 0}$ (MID)

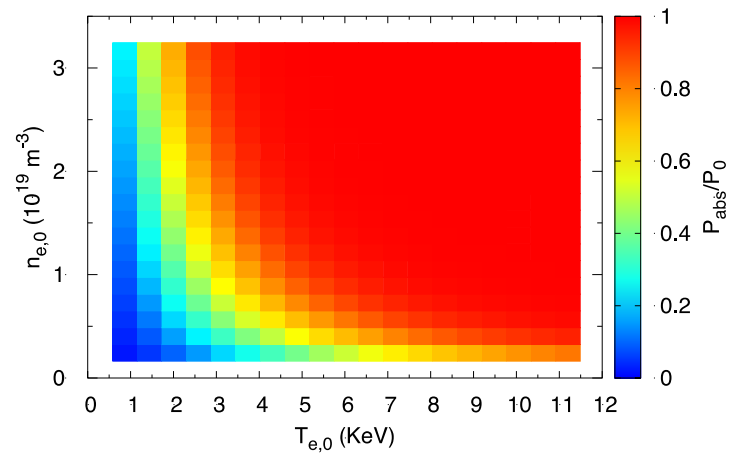

Fig. 4b. Absorbed efficiency as a function of $\mathrm{T}_{\mathrm{e}, 0}, \mathrm{n}_{\mathrm{e}, 0}$ (USM).

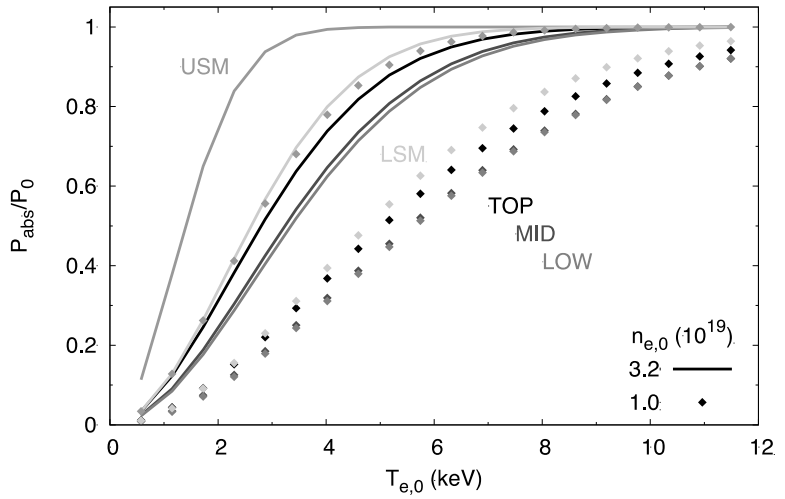

Fig. 5. Absorbed efficiency as a function of $T_{e, 0}$ for different $n_{e, 0}$ scaling; lines correspond to slices at constant $n_{e, 0}$ in Fig.4a,b and equivalent maps for other mirrors.

As reported in Table 2, a good absorption efficiency (90\% of input power absorbed) can be reached only for $\mathrm{T}_{\mathrm{e}, 0} \geq 5 \mathrm{keV}$ for EL mirrors and around this value for LSM. Only USM allows to reach a good absorption already at $\mathrm{T}_{\mathrm{e}, 0} \geq 3 \mathrm{keV}$ for $\mathrm{n}_{\mathrm{e}, 0} \geq 2 \cdot 10^{19} \mathrm{~m}^{-3}$, but the threshold temperature increases rapidly with decreasing density. This mirror alone, however, would allow the use of only 16 of the 24 available beams (13.3 MW out of 20).

Table 2. $\mathrm{T}_{\mathrm{e}, 0}$ for $90 \%$ absorption for different density scaling.

\begin{tabular}{ccc} 
& \multicolumn{2}{c}{$\mathbf{n}_{\mathbf{e}, \mathbf{0}}\left(\mathbf{m}^{-\mathbf{3}}\right)$} \\
& $\mathbf{3 . 2 \cdot 1 \mathbf { 1 0 } ^ { \mathbf { 1 9 } }}$ & $\mathbf{1 . 0 \cdot 1 0 ^ { \mathbf { 1 9 } }}$ \\
\hline TOP & $5.5 \mathrm{keV}$ & $10.2 \mathrm{keV}$ \\
\hline MID & $6.2 \mathrm{keV}$ & $10.9 \mathrm{keV}$ \\
\hline LOW & $6.4 \mathrm{keV}$ & $10.9 \mathrm{keV}$ \\
\hline USM & $2.7 \mathrm{keV}$ & $5.1 \mathrm{keV}$ \\
\hline LSM & $4.9 \mathrm{keV}$ & $9.2 \mathrm{keV}$ \\
\hline
\end{tabular}

The difference in efficiency between MID/LOW and TOP, is due to the different toroidal injection angles. Beams from UL mirrors are more focused than those from EL and are nearly tangent to the magnetic surfaces, being able to travel for a longer path in the resonance region. Moreover, USM is able to reach deeper into the plasma thanks to its higher poloidal angle.

The low absorption efficiency for low temperature values means that only a fraction of the injected power is available for heating, thus leaving a relevant amount of residual power free to reach the wall on the HFS of the machine, where it is reflected and partially converted to O-mode polarization. This fraction, around $20 \%$ of incident power, is then able to pass through the plasma undergoing minimal absorption and, for beam originating from EL mirrors, can possibly reach the LFS wall and ports.

\subsection{Power deposition profiles and CD}

The presence of the $n=4$ harmonic inside the plasma can impact on the accessibility to the plasma inner regions and also on the Current Drive (CD) efficiency, although if the goal is pure heating a low CD is not an issue. The power deposition profiles, as shown in Fig. 6, indicate that the deposition region for the UL mirrors are significantly narrower $\left(\Delta \rho_{\text {tor }}<0.1\right)$ than for those of EL. This is due to the fact that the beams propagating form UL mirrors enter the plasma already behind the $n=4$ harmonic resonance and do not interact with it.

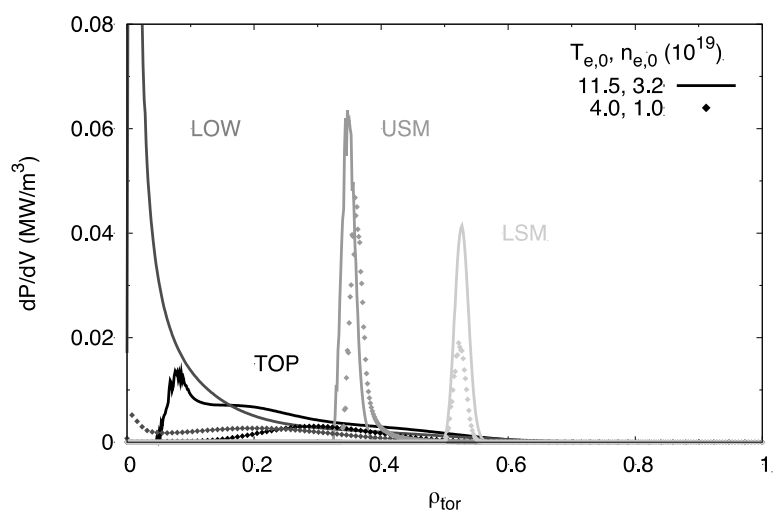

Fig. 6. Power deposition profile for different $T_{e}, n_{e}$ scaling $\left(T_{e}, 0\right.$ in $\mathrm{keV}, \mathrm{n}_{\mathrm{e}, 0}$ in $\mathrm{m}^{-3}$ ). 
The fact that the beams are nearly tangent to the magnetic surfaces in the region of $n=3$ harmonic resonance means that the spreading of the power deposition region depends more on the local beam width than density and temperature scaling, which impact on it mainly through their effect on local optical thickness. The beams launched form EL mirrors, conversely, cross both $n=4$ and $n=3$ harmonic resonance regions and are nearly perpendicular to the magnetic surfaces, presenting broader profiles. EL mirrors however are able to reach regions of plasma at lower radii than UL, that is restricted to $\rho_{\text {tor }}>0.3-0.5$. The interaction of $n=4$ harmonic with the EL mirrors becomes evident when evaluating the fraction of power it absorbs compared to the total absorbed power, as shown in Fig. 7a,b.

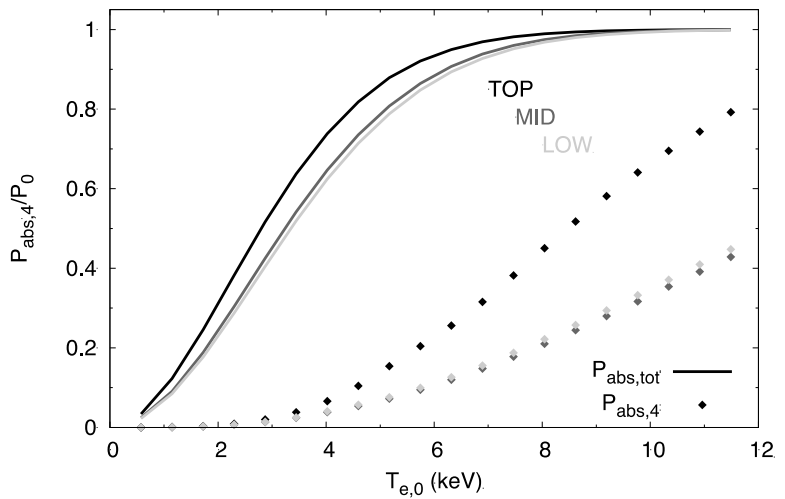

Fig. 7a. Total and power absorbed by $\mathrm{n}=4$ harmonic resonance for $\mathrm{n}_{\mathrm{e}, 0}=3.2 \cdot 10^{19} \mathrm{~m}^{-3}$; solid lines corresponds to lines in Fig. 5 .

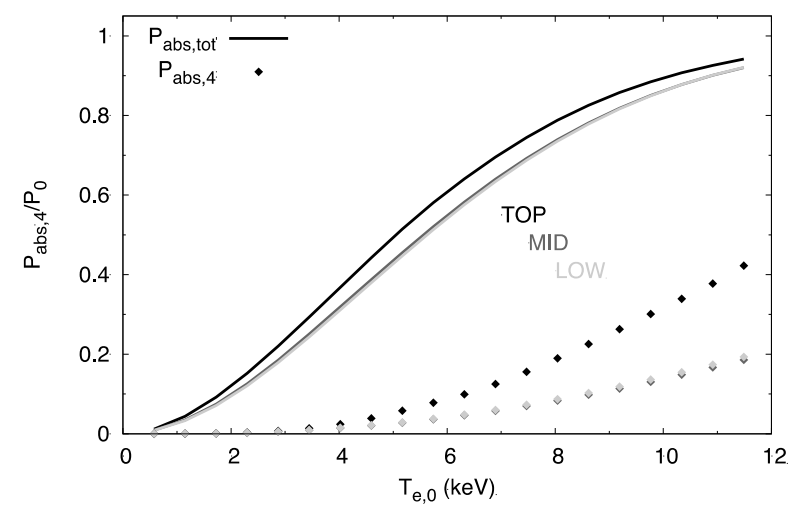

Fig. 7b. Total and power absorbed by $\mathrm{n}=4$ harmonic resonance for $\mathrm{n}_{\mathrm{e}, 0}=1.0 \cdot 10^{19} \mathrm{~m}^{-3}$; solid lines corresponds to dots in Fig. 5 .

The absorption for EL mirrors can become significant with increasing $\mathrm{T}_{\mathrm{e}}$. The most affected is the TOP mirror, for which $\mathrm{P}_{\mathrm{abs}, 4} / \mathrm{P}_{0} \geq 50 \%$ for $\mathrm{T}_{\mathrm{e}, 0}>8.5 \mathrm{keV}$.

This might be an issue for Current Drive applications. The $n=4$ harmonic absorption, in fact, has two detrimental effects on the driven current $\left(\mathrm{I}_{\mathrm{CD}}\right)$ : it reduces the power that can reach the higher efficient $n=3$ harmonic resonance; it generates a current flowing in the opposite direction of the $I_{C D}$ generated by $n=3$. Its $C D$ efficiency, however, is very low compared to $n=3$ harmonic and thus the opposite $\mathrm{I}_{\mathrm{CD}}$ is very low or negligible.

The reduction of power reaching the lower harmonic can have a greater effect: both $n=3$ harmonic $C D$ efficiency and $n=4$ harmonic power absorption efficiency increase with $T_{e}$. At high $T_{e}$, if the $n=3 C D$ efficiency is not able to compensate the loss of power due to $n=4$ absorption, the $I_{C D}$ it generates can decrease, as shown in Fig. 8 for the LOW and TOP mirrors (similar results for MID) starting from $\mathrm{T}_{\mathrm{e}, 0} \sim 8-9 \mathrm{keV}$.

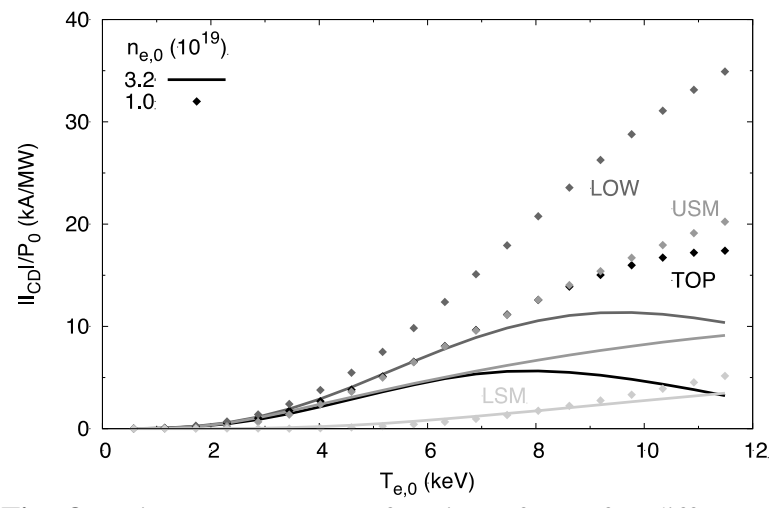

Fig. 8. Driven current as a function of $T_{e, 0}$ for different $n_{e}$ scaling $\left(\mathrm{n}_{\mathrm{e}, 0}\right.$ in $\left.\mathrm{m}^{-3}\right)$.

\section{Performances at 104}

\subsection{Power absorption efficiency}

The same analysis described in the previous section has been performed for the three EL mirrors simulating the propagation of X-mode polarized beams with $\mathrm{f}=104$ $\mathrm{GHz}$ (the use of dual-frequency gyrotrons is foreseen, if realized, only for the three EL mirrors). The beam parameters are those used in the previous section, since a design of an EC system operating at $\mathrm{f}=104 \mathrm{GHz}$ is not available at the moment.

The use of the lower frequency, as said before, would allow to take advantage of the $n=2$ harmonic resonance, which for $\mathrm{B}_{0}=1.8 \mathrm{~T}$ and $\mathrm{f}=104 \mathrm{GHz}$ is located on the HFS near the centre of the plasma. For this part of the analysis the temperature scaling has been extended down to 0.005 times the unscaled profile $\left(\mathrm{T}_{\mathrm{e}, 0} \sim 50 \mathrm{eV}\right)$, in order to identify the range of $\mathrm{T}_{\mathrm{e}}, \mathrm{n}_{\mathrm{e}}$ values for which the EC waves are not completely absorbed on the first pass in the plasma. In fact, the simulations have shown a complete absorption of the injected power for all but the lowest density and temperature values in the previously used scaling range, as can be guessed from Fig. 9.

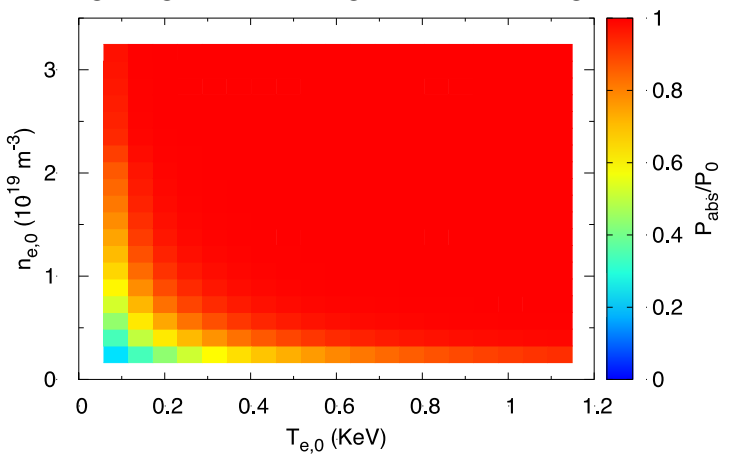

Fig. 9. Absorbed power fraction as a function of $T_{e, 0}$ and $n_{e, 0}$ for TOP mirror; similar results are found for MID and LOW.

The $\alpha_{\text {opt }}$ values determined for $\mathrm{f}=170 \mathrm{GHz}$ have been used also for the lower frequency. Using $104 \mathrm{GHz}$ EC waves would thus allow full absorption even for temperature values found in an ohmic plasma, with no 
risk of residual power propagating around the vessel. At high density there can be a decrease in absorption due to refraction effects, especially for beams not pointing towards the plasma centre $\left(\mathrm{n}_{\mathrm{e}, 0} / \mathrm{n}_{\text {cut-off }} \sim 0.5\right.$ for the highest density value considered in this analysis).

\subsection{Power deposition profiles and CD}

The $n=3$ harmonic is located outside the plasma, and shouldn't therefore have a significant interaction with the injected beams. The analysis shows that there can be a small fraction of injected power absorbed by $n=3$ harmonic resonance, due to high energy electrons, but it always remains under $5 \%$ of total absorbed power, even for the highest temperature values considered. Fig. 10 shows the power density absorption profile for the different EL mirrors, similarly as Fig. 6. As for $\mathrm{f}=$ $170 \mathrm{GHz}$, the profiles are still wider than UL, albeit narrower than for the higher frequency $\left(\Delta \rho_{\text {tor }} \sim 0.1-0.15\right)$ due to the fact that only one harmonic resonance is involved. As in Fig. 6, it is clear that density and temperature scaling does not impact significantly on the power deposition width while does influence the radial deposition location, and that the EC waves are still able to reach the inner regions of the plasma.

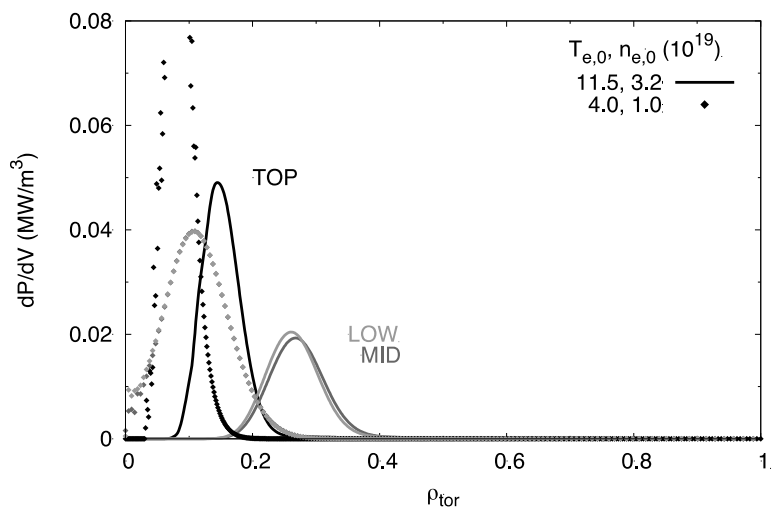

Fig. 10. Power deposition profile for different $T_{e, 0}, n_{e}$ scaling $\left(\mathrm{T}_{\mathrm{e}, 0}\right.$ in $\mathrm{keV}, \mathrm{n}_{\mathrm{e}, 0}$ in $\left.\mathrm{m}^{-3}\right)$.

The $n=2$ harmonic resonance higher Current Drive efficiency in conjunction with the higher absorption result in a higher driven current than $f=170 \mathrm{GHz}$ case, as shown in Fig. 11.

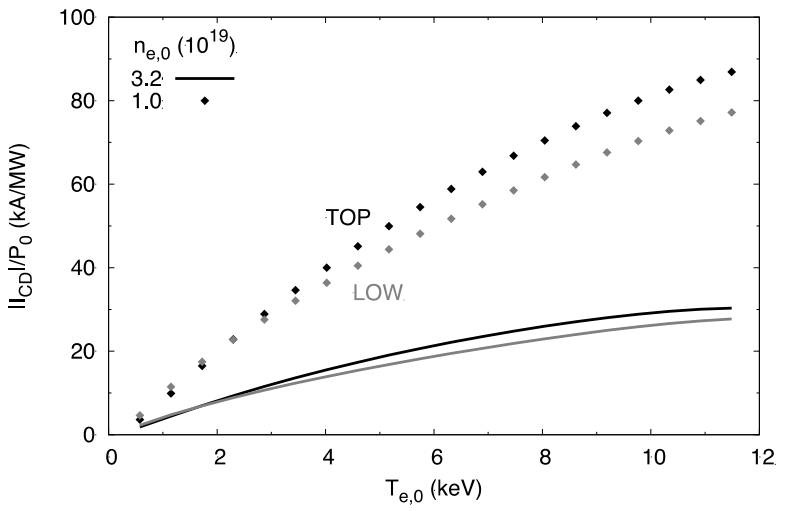

Fig. 11. Driven current as a function of $T_{e, 0}$ for different $n_{e}$ scaling $\left(\mathrm{n}_{\mathrm{e}, 0}\right.$ in $\left.\mathrm{m}^{-3}\right)$.

For $\mathrm{f}=104 \mathrm{GHz}$ only one harmonic resonance $(\mathrm{n}=2)$ contribute significantly to absorption. This avoids the effects causing a decrease in $\mathrm{I}_{\mathrm{CD}}$ at high temperatures, unlike the higher frequency.

\section{Conclusions}

This study provided a preliminary estimation of the performances of an ECRF system using the two frequencies in a one-third magnetic field scenario.

The analysis performed has shown that the $170 \mathrm{GHz}$ ECRF system of ITER can ensure good absorption efficiency also for one-third field operations, provided the electron temperature is higher than approximately 5 $\mathrm{keV}$ for typical density values of the order of $3 \cdot 10^{19} \mathrm{~m}^{-3}$. At lower temperature, the unabsorbed fraction of the injected power shines through the plasma hitting the HFS wall and can potentially reach the LFS wall and ports. The only exception is the USM, which already shows good absorption at lower temperature $\left(\mathrm{T}_{\mathrm{e}, 0} \geq 3\right.$ $\mathrm{keV}$ for $\mathrm{n}_{\mathrm{e}, 0} \geq 2 \cdot 10^{19} \mathrm{~m}^{-3}$ ), easily reachable with ohmic heating. This means that at least 13.3 MW out of the 20 MW available to the EC system can be used to help raising the temperature to the point where the other launchers are effective.

The use of dual-frequency gyrotrons operating also at $104 \mathrm{GHz}$ would allow to obtain a complete power absorption starting from much lower temperature and density values, allowing to bridge the gap between the ohmic plasma conditions at the beginning of the discharge and the range of efficiency of the $170 \mathrm{GHz}$ system.

The use of $\mathrm{f}=104 \mathrm{GHz}$ would also allow a higher Current Drive efficiency with respect to $\mathrm{f}=170 \mathrm{GHz}$, which has limited $\mathrm{CD}$ efficiency, especially for EL mirrors at high temperature $\left(\mathrm{T}_{\mathrm{e}, 0} \geq 8-9 \mathrm{keV}\right.$ for $\mathrm{n}_{\mathrm{e}, 0} \geq$ $3 \cdot 10^{19} \mathrm{~m}^{-3}$ ), due to the presence of $\mathrm{n}=4$ harmonic in the LFS of the plasma

A complete assessment of the performance would need a more in-depth analysis, accounting for mutual influence between plasma profiles evolution and EC power absorption.

\section{References}

1. M. Henderson, et al, Phys. Plasmas 22, 021808 (2015)

2. Y.R. Martin, et al, Journal of Physics : conference Series 123, 012033 (2008)

3. V. Parail, et al, Nucl. Fusion 53, 113002 (2013)

4. D. Farina, Fusion Sci. Technol. 52, 154 (2007) 